of this intervention, multi-professional teaching regarding changes to e-handover was provided, with the intention to engage the whole team in supporting communication within and between professional groups, and to improve patient care. Methods A survey was designed to measure staff views on their perceived impact of the SOP on the handover, communication and patient care. This was administered over a two week period in September 2017 on paper and electronically. Twenty-seven surveys were completed by doctors, nurses and clinical support workers who use e-handover.

Results Ninety-two percent of those surveyed felt that the handover was easier to read, $100 \%$ felt they had a clearer understanding of what information to include on the handover, $92 \%$ had a better understanding of escalation status and $47 \%$ of nurses felt it was easier to explain this status to a doctor out of hours. Assessing advance care planning, 85\% felt they had a better understanding of a patient's preferred place of death. Sixty-six percent of those surveyed felt patient care had improved as a result of these changes. Comments included 'this has been a great development which ensures a more robust and consistent approach to handover. However, $48 \%$ felt the changes had resulted in handover being more time consuming.

Conclusion Multi-professional input to support changes in the e-handover led to improved communication between staff, a clearer understanding of escalation status and preferred place of death for patients, leading to improved patient care. Further work is required to understand the cause of time burdens in completing handover.

\section{A TALE OF TWO SETTINGS: ARE THERE DIFFERENCES IN HOSPICE INPATIENT INITIAL NEEDS \& OUTCOMES DEPENDING ON WHERE THE PATIENT IS ADMITTED FROM?}

FA Malik, S Clarke, D Barclay. St. Wilfrid's Hospice, Eastbourne, East Sussex NHS Healthcare Trust

\subsection{6/bmjspcare-2018-ASPabstracts. 162}

Introduction Understanding patient needs and assessing outcomes are important to improve quality of care. As part of an ongoing evaluation of hospice inpatient (IPU) bed use, the needs \& outcomes of those admitted to the IPU from the local hospital trust and from community settings were compared to examine if there were differences depending on settings admitted from.

Methods Cross-sectional retrospective analysis of consecutive IPU admissions from the local hospital NHS Trust (HA) and admissions from the community (CA) to a specialist palliative 14-bedded hospice from August 16-March 17. Data items collected include patient demographics \& outcome data e.g. IPU admission IPOS, phase of illness (PoI) \& AKPS. All data anonymised. Results analyzed and descriptive statistics utilized.

Results $50 \mathrm{HA}$ and $113 \mathrm{CA}$ admissions during the time period (in addition 24 respite admissions and 6 'out of area' HA not used in analysis). There were no differences in mean age (HA $71.3 \mathrm{yrs}, \mathrm{CA} 71 \mathrm{yrs}$ ) or gender (HA 60\%, CA 53\% male) and no difference in malignant diagnosis between groups (HA $82 \%$, CA $88 \%$ ).

IPU admission mean AKPS was worse in HA (33) than CA (44.6) $(\mathrm{p}<0.005)$ but no difference in mean IPOS (HA 31.2, CA $34, \mathrm{p}=0.1)$.
HA patients were more likely to be in the 'dying phase' (PoI) on IPU admission (14/50,28\%) compared to other phases than CA patients $(9 / 110,8 \%)(\mathrm{p}<0.001)$. Mean IPU length of stay was longer in CA (17.6 days) compared to HA (13.2 days) $(p=0.05)$. HA were more likely to die during that IPU admission compared to CA patients (46/50 (92\%) vs $69 / 110$ $(62 \%)),(p<0.05) .41 / 110$ CA patients were discharged from IPU.

Conclusions Findings highlight potential differences in patient needs and outcomes depending on which setting the patient was admitted from. This will influence development of 24/7 services $\&$ bed utilization. Further analysis is beneficial to clarify findings.

\section{CARE OF PATIENTS WITH PROGRESSIVE SUPRANUCLEAR PALSY IN THE HOSPICE SETTING: AN AUDIT}

Kate Hoddell, Laura Dewhirst, Derek Willis. Severn Hospice

\subsection{6/bmjspcare-2018-ASPabstracts. 163}

Hospices are increasingly focused on improving services for patients with life-limiting non-malignant conditions. Progressive supranuclear palsy (PSP) is a life-limiting progressive neurological disease which can be mistaken for Parkinson's disease early in its course. Symptoms progress rapidly within 5-7 years. Patients with PSP may have a high number of symptoms and will have a poor prognosis.

This retrospective audit aimed to evaluate care provided to patients with PSP referred to Severn Hospice using the guide 'Pathway of Care for PSP: A guide for Health and Social Care Professionals' as the standard. It included all patients under hospice care since 2015.

20 patients were included, 8 of whom continued under hospice care. 7 patients had died and 5 discharged. Time between diagnosis and referral to our service was usually in the first year. Most patients were referred for 'medical assessment and support' and had more than 3 symptoms at referral. 5 patients were admitted to the hospice, 3 of whom died during admission. These patients had at least 4 symptoms at referral.

7 patients had no advance care planning (ACP) completed (defined as a do not resuscitate order (DNAR), preferred place of care (PPC), preferred place of death (PPD) and/or an advance decision to refuse treatment (ADRT)). Of the other patients 13 had a DNAR, 5 had a documented PPC, 5 had a documented PPD and 2 patients had an ADRT. Of the 7 who died, only 2 had a PPD; both achieved their PPD.

This audit showed that while this is a small number of patients, this is a group with a high symptom burden at referral to palliative services. This suggests that palliative medicine has a role early in the patient journey. ACP was not fully completed in any audited patients indicating that this is an area requiring improvement.

\section{IMPROVING ADVANCE CARE PLANNING IN A TERTIARY ONCOLOGY CENTRE}

Harriet Radcliffe, Lori Low, Kiara Parag, Rachel Marshall, Aravind Parkash, Eleanor Smith, Alice Dewdney. Sheffield Teaching Hospitals Foundation Trust

10.1136/bmjspcare-2018-ASPabstracts. 164 